\title{
Magnetic Water Treatment in Environmental Management A Review of the Recent Advances and Future Perspectives
}

\author{
YADOLLAHPOUR ALI*, RASHIDI SAMANEH, REZAEE ZOHRE and JALILIFAR MOSTAFA \\ Department of Medical Physics, School of Medicine, Ahvaz Jundishapur University \\ of Medical Sciences, Golestan Blvd., Ahvaz, Iran.
}

http://dx.doi.org/10.12944/CWE.9.3.56

(Received: December 06, 2014; Accepted: December 23, 2014)

\begin{abstract}
Magnetic water treatment (MWT) is a relatively new technique in environmental management. Magnetic field exposure alters physical and chemical properties of water molecules resulting in unique characteristics. Magnetized water has shown various properties with possible applications in different fields of environmental management. Scale prevention/elimination, soil enhancement, plant growth, crop yield, water saving, and wastewater treatment are some of these applications. Magnetic treatment of water restructures the water molecules into tiny, uniform and hexagonally structured cluster easing their travel through the passageways in plant and animal cell membranes. In addition, toxic agents cannot enter the MW structure. These features make MW a bio-friendly compound for plant and animal cells. The present study reviews the applications of MWT in environmental management. The recent advances of MWT in different fields of environment management are discussed. In addition, the mechanism of action of MWT in different applications and future perspectives are discussed.
\end{abstract}

Key words: Magnetic Water Technology, Scale prevention, Electromagnetic field.

\section{INTRODUCTION}

Recent decades have witnessed a dramatic increase in the applications of electromagnetic fields (EMFs) in different domains including therapeutic and diagnostic medicine, environmental managements and industrial procedures[1-7]. Magnetic water treatment (MWT) is one these applications with promising potentials in health, industry and environment. Water is a crucial source for life and any living creature needs it to hydrate every cell. Long term and frequent droughts and competing water demands in most parts of the world have caused severe pressure on water resources. In addition, high cost of irrigation worldwideis the main problem of agriculture development. Therefore, developing newtechniques for water consumption reductionhas drawn research interest of scientists worldwide. Magnetic water technology is a noble approach for water treatment to achieve different potentials [8]. Various studies have revealed promising potentials of magnetic treatment in different fields of environmental management. The beneficial effects of magnetic treatment in farming and agriculture, food processing, wastewater treatment and scale prevention/elimination have been highlighted in different studies. These effects can be categorized into two main groups: the effects of magnetic water treatment on different living organisms and objects; and the direct effects of magnetic treatment on the organisms and objects. The main applications of MWT in farming and agriculture include improvement of irrigation water quality and crop yield, water distribution scale elimination/prevention, soil improvement, corrosion control and wastewater treatment[9, 10]. In addition, the antimicrobial and antiseptic effects of MF are used in wastewater treatment and food and fruit juice processing and homogenization[1115]. Magnetized or magnetic water (MW) can 
prevent scale formation and eliminate scale from the surface of pipeline and water-using equipments. Similarly, magnetic treatment of fuel can improve the engine performance and save fuel consumption through elimination and prevention of scale[16, 17] [6]. The present study reviews the basic principles of MW technology and its current applications in environmental management, their advantages and recent advances in each field.

\section{Magnetic Water Treatment}

MWT is a simple and efficient approach where the water flows through an MF or combination of MFs which consequently acquires different physicochemical characteristics. Water is a paramagnetic compound with small and positive susceptibility to MFs. The water molecules like other paramagnetic compounds are slightly attracted by an MF and the material does not retain the magnetic properties when the external field is removed $[18,19]$. When passing under an MF, water gains a magnetic moment that persists for 24 to $48 \mathrm{hrs}$. The outcome of MWT depends on the MF intensity, composition of dissolved salts and velocity of crossing a magnetron of 0.5 inch diameter. MWT is a non-chemical treatment of water that does not require any filtration substitutes. In agriculture maximum, healthy and efficient hydration is achievable with MW. MF can influence the structures of cell membrane, their permeability, ion transport and therefore effect on metabolic pathways of them [20]. MF treatments through stimulating the activity of proteins and enzymes can influence free radicals and overall in biochemical processes [21, 22]. In normal or non$\mathrm{MW}$, the water molecule clusters comprising of many water molecules are loosely attracted. This loose and chaotic form of attraction predisposes the water to toxins and pollutants to travel inside the water molecule cluster. The large structure of these water molecule clusters or presence of toxins blocks large portions of these clusters when they pass through the cell membrane. The smaller size of these chaotic clusters, some of them carrying toxins, can enter the cell with consequent harmful effects [22]. Therefore, to hydrate a plant a great deal of normal water is required. Magnetic treatment of water redirect the water molecules into very small clusters, each made up of six symmetrically organized molecules. This tiny and uniform cluster has hexagonal structure thus it can easily enter the passageways in plant and animal cell membranes. In addition, toxic agents cannot enter the MW structure. These features make MW a bio-friendly compound for plant and animal cells. MW can be used to increase crop yield, induce seed germination and benefit the health of livestock. Studies have demonstrated that MW for irrigation can improve water productivity [10]; thus, conserving water supplies for the expected future global water scarcity. In addition, MW is reportedly effective at preventing and removing scale deposits in pipes and water containing structures. MW water enhances the levels of $\mathrm{CO} 2$ and $\mathrm{H}+$ in soils comparable to the addition of fertilizers. Combining cleaning agents with MWT increases the effectiveness of the agents so that the cleaner consumption by one-third to onefourth $[23,24]$.

\section{Magnetic Water Treatment in Agriculture}

Natural water contains micro and macroparticles of organic and inorganic natures along with different ions, phytoplankton, and microbubbles [25]. In normal or non-MW, the water molecule clusters are loosely attracted. These clusters are in different sizes depending on the number of water molecules. This loose and chaotic form of attraction increases the bonding of toxic agents and pollutants. A small fraction of these large structure clusters can enter the cell membrane. In addition, toxic agents can adhere to this loosely structured water cluster molecules which consequently can enter the cell membrane with adverse effects [22]. Therefore, to hydrate a plant a great deal of normal water is required. MWT process is based on the magnetohydrodynamics principle, where MF exerts and adds electrical energy to charged particles in water that contains ions and small solid particles with electrostatic charges. In MWT, water passes through a magnetic field or combination of magnetic arrays where a Lorentz force is exerted on each ion which is in the opposite direction of each other. The redirection of the particles increases the frequency of collisions between ions of opposite sides, combining to form a mineral precipitate or insoluble compound. In addition, toxic agents cannot enter the MW structure. These features make MW a bio-friendly compound for plant and animal cells[19, 26-28].

MW can be used to increase crop yield, induce seed germination and benefit the health of livestock. Studies have demonstrated that using 
MW for irrigation can improve water productivity. In addition, MW is reportedly effective at preventing and removing scale deposits in pipes and water containing structures[9, 29-39].

\section{Magnetic Wastewater Treatment}

EMFs have shown promising potentials for water and wastewater treatments (WWT). Safety, compatibility and simplicity, environmentally friendliness, low operating cost and not proven harmful effects are the main advantages of EMFs in these fields[6, 16, 17, 32, 35, 40-45]. In addition to the antimicrobial and antibacterial effects of EMFs on wastewater treatment, these fields have different characteristics which can be used to enhance a WWT process: modifying the physical and chemical properties of water molecules and other elements, precipitating sludge, removing phosphorus and organic compounds from solutions. Combining cleaning agents with MWT increases the effectiveness of the agents so that the cleaner consumption by one-third to one-fourth [23]. Previous laboratory and field studies have shown the high efficacy of EMFs as adjunctive or alternative treatment for conventional WWT. Different modalities of EMFs can improve different steps of WWT process: EMFs can improve the activating sludge and disinfection processes [17, 32, 43,44]. In addition, antimicrobial and antibacterial properties of different frequency-bands of EMF spectrum like ultraviolet (UV) radiation are useful in the WWT [3, 16, 32, 40,43-46]. These antimicrobial effects can improve the disinfection process of the WWT process or they can be used as a stand-alone wastewater treatment process. Furthermore, EMFs can alter physical and chemical properties of water molecules, microorganisms and organic compounds that will be used to facilitate sewage treatment process [32, $43,46]$. MFs can modify the activity of activated sludge that is a crucial factor in the WWT process [17, $32,44]$.

The main research fields of EMF applications in WWT are magnetite slurry, magnetic particles, pulsed electric field (PEF), pulsed EMF, altering activated sludge, and magnetic powder. Majority of magnetic WWT systems are similar in using magnets or electromagnets as the core element of a complete system to eliminate pollutants from wastewater including phosphate, heavy metals organic ions. Magnetic particle technology is one of the common magnetic WWT method and acts on the basis of absorption and coagulation process [16]. This technique can be used for various wastewater treatment processes such as metal recovery from electroplating rinse water, sewage sludge and hydrometallurgical effluents. This process is based on attachment of wastewater pollutants to a magnetic carrier material (magnetite). After separation, the magnetite is recovered and reused in the process. The other EMF-based technique for WWT is static MF which has attracted a considerable research attention. Little well documented scientific data is available on the effect of SMF on biodegradation process of wastewater organic substrates, especially transformations of nitrogen compounds.

\section{Technical Considerations and Mechanism of Action}

The effectiveness of MWT in wastewater treatment, scale elimination/ prevention and increasing biodegradability of microorganisms is strongly affected by the chemical properties of the treated medium, strength and configuration of the MF, thermodynamic properties of the water and fluid flow characteristics. In MWT, to achieve desired outcomes, the four important conditions should be observed: a) The water path must be perpendicular to the magnetic lines of force; b)Water should first cross the south magnetic lines and then continue to break wider and denser alternating reversing polarity lines, until leaving the magnetic chamber through the single North Pole flux path; c) The capacity of a magnetic conditioner can be determined by the gauss strength, flux density, area surface of the exposure of the number of fields and the distance between alternating poles; d) Water should be under pressure and flowing with as minimum turbulence as possible, just before entering and during its travel through the MFs.

Biological processes are used to convert organic materials existed in wastewater into flocculant and settleable biological and inorganic solids. Under these processes, the biodegradability of sludge is increased. Activated sludge process and its modified forms are among the most commonly used biological processes[17, 32, 43, 44]. The effects of MFs on living organisms have been usually conflicting. Although some studies have 
reported negative impacts of MFs on the growth of living organisms, most of them have shown enhancement effects. The MF-induced effects on the living organisms depend on the strength of MF and the exposed microorganism type. Although the exact mechanisms of action of these effects have not been understood in detail, it is obvious that MF has not a lethal effect on microorganism. MF exposure can increase or decrease the growth rate of a living organism depending on the physical parameters of the field like type of magnetic pole, South pole or North Pole, exposure duration, intensity,etc. Goodman et al. evaluated the effects of weak pulsed MFs (1.5 mT peak ,2s on 2s off) on Escherichia coli strains. Their results showed that the synthesis of numerous proteins are either increased or decreased by a factor of 2 or more. Tabrah et al. indicated that 48-h exposure to a $60 \mathrm{~Hz}$ sinusoidal EMF (intensity of $0.2 \mathrm{mT}$ ) significantly increased the number of azide induced TA100 revertant colonies of Salmonella typhimurium.

The appropriate strength of MF to improve biological process of WWT is not yet determined. The MF induction reported by previous studies is wide and ranges 7 to $490 \mathrm{mT}[17,30,38,39,47]$. Jung and Sofer[38] showed that application of MF at induction of 150 and $350 \mathrm{mT}$ improved phenol biodegradation through immobilized activated sludge. They used unipolar South Pole of MF. However, in the earlier studies Jung et al. [39] showed the positive effect of MF on phenol biodegradation but at induction of $490 \mathrm{mT}$.Yavuz and Çelebi[17] demonstrated that MF of $17.8 \mathrm{mT}$ induction generated by direct current supplied solenoid supported glucose biodegradation with activated sludge action. Cebkowska[47] showed that static MF at induction range of 5 to $140 \mathrm{mT}$ generated by electromagnet coils intensifies the biodegradation process by activating sludge for majority of testing organic substrates. The main issue in the development of commercial systems of wastewater treatment based on EMFs is conducting cost analysis of experimental systems and designing cost-effective and practical systems with reasonable cost.

\section{Antimicrobial effects of EMF}

EMFs have shown antimicrobial effects in different frequencies and intensities. A detailed review of the recent applications of EMFs as antimicrobial and antibacterial treatment and the proposed mechanisms of actions is provided by Yadollahpour et al. (2014)[3].Different modalities of EMFs showed antimicrobial and antibacterial effects in different pathogens. Electric fields, magnetic fields and pulsed EMFs (PEMFs) are common techniques showing promising antimicrobial effects. Despite the various studies indicating the antimicrobial effects of EMFs, the mechanisms of actions of them are not yet completely understood. Systematic review of studies published on the antimicrobial effects of EMFs showed that static MF and PEMFs show promising antimicrobial effects for some of common bacterial pathogens. These treatments can be developed as alternative or at least as an adjunctive treatment for some infectious diseases and chronic wound[2].Static MFs showed inhibiting effects over different bacterial pathogens. One of the theories that can explain the inhibiting and fatal effects of SMFs on living organisms and cells is membrane theory [48-53]. This theory explains that the diamagnetic properties of membrane phospholipids determine the effects of SMF on living organisms. The reorientation of these molecules during SMF exposure results in the deformation of imbedded ion channels. This deformation alters the induced rotational excitation of the hydrocarbon chain which eases the reorientation of the molecules. Therefore, the effects of SMFs on organisms are enhanced. The main conclusion from the previous studies on the effects of electric and magnetic fields on living microorganisms is that some modalities of the EMFs inhibit the activities of some pathogens, while excite other pathogens. Further controlled studies are needed to develop new antimicrobial techniques based on EMFs.

\section{Scale Prevention and Elimination}

Chemical scaling inhibitors change the water composition; thus, they have to be avoided for drinking water distribution. Therefore, physical treatments are good candidate for scale prevention/ elimination because they do not change the potability of water.

Suspended particles or solids in water can cause serious problems in irrigation distribution systems and also drink water networks. The deposition of scale can even completely block an irrigation system. Therefore, an effective water treatment is necessary which should be cost 
effective while insuring the minimum environmental pollution. Water used in industry has different origins including rivers, lakes, wells and oceans; it always contains dissolved and suspended solids. The hardness of water is classified as temporary and permanent. Temporary hardness is because of dissolved bicarbonates in the water, mainly calcium and magnesium that can easily be removed by heating to break them down into carbonates which are insoluble and precipitate out. Permanent hardness, induced by soluble sulfates and chlorides of calcium and magnesium, cannot be made insoluble by heating. Suspended particles are also the origin of problems for water distribution systems or fuel engines. These particles can be separated by physical approaches such as centrifuging, settling or hydrocycloning. Suspended solids present in water such as sand, clay, silt and corrosion products are incorporated into scale together with micro-biological pollutants and corrosion impurities. The deposition of scale makes it necessary to shut down water-using equipment at intervals so that the build-up can be removed. Sometimes, this shutdown will be part of a planned maintenance program. However, the progression of scale can severely or even completely block the system, making it necessary to remove the equipment from service to remove the blockage. Scale deposits can also increase corrosion, due to entrapped oxygen and corrosive because the scale itself is some-times to the surface with which it is in contact. Furthermore, scale will disturb the action of inhibitors in the system, making a barrier between these agents and the scale beneath surface. If the scale is patchy, the differential aeration between the clean and scaled surface facilitates the built up of a corrosion cell. To prevent scale formation several nonchemical methods of water treatment that are based on permanent magnetic, electromagnetic, electric, electrostatic, ultra-sonic, radiation have been introduced [54-57]. One of the promising applications of MWT is to suppress water-scale deposition on the inner surface of boilers, heat exchangers and pipelines. MFs in appropriate spatial and intensity conditions can modulate the turbulence in the fluid. Magnetically structured water can remove scaling from water distribution system and also prevents new scaling [54-57]. In agriculture and farming, magnetized water for irrigation can save irrigation water [58]. In environmental managements, scale prevention/ elimination by magnetic treatment can reduce the energy consumption and air pollutions. In industries, engine with higher performance, fuel saving are the main benefits of magnetic treatment.

The rate of three dynamic phenomenon play important roles in the crystallization process: association, dissociation and nucleation rates. Different studies have shown that under certain conditions in the presence of an external MF, the nucleation rate can be greatly increased. This was observed as a rapid onset or "burst" of crystallization within the bulk solution, and can be quantitatively described as the scattering of incident light-the so-called Tyndall effect[59]. As a consequence of the faster precipitation in the presence of the magnetic field, the resultant crystals are greater in number, with smaller sizes and irregular shapes [59][25, 37, 59].

Laboratory studies have indicated that magnetohydrodynamic effects are probably responsible for scale prevention/elimination traits of magnetic treatment of water. Magnetic treatment devices are effective for scale control in waterusing systems. In addition to enhancing corrosion of metals in the vicinity of the device, or within the device itself, application of the field $90^{\circ}$ to the flow of a conducting fluid can alter the hydrodynamics of fluid flow. Depending on experimental conditions, this may increase or decrease turbulence in the fluid, promoting aggregation or deaggregation of both ferromagnetic and diamagnetic colloids[57]. Important factors influencing the magnetohydrodynamic forces on fluid flow are conductivity of the solution, linear flow velocity of the fluid, and the flux density (magnetic induction) of the transverse field. Magnetic treatment devices that are physically designed to create additional turbulence by constricting or otherwise altering fluid flow may further enhance the antiscaling effect by purely mechanical means[57][59] $[25,37]$.

\section{Magnetic water treatment in fuel performance}

When any heat-transfer surface becomes scaled, the insulating scale reduces the efficiency of the equipment, increases fuel requirements and maintenance. Some studies have claimed the effectiveness of MFs in fuel performance. The main mechanisms of this enhancement are scale 
prevention/elimination within the engine and different fuel-containing parts and increasing the combustion ratio[18, 37, 43, 57, 60, 61].

Some studies have shown that inserting a small, powerful magnet on the fuel line of an internal combustion engine increases fuel mileage about 5 to $15 \%$. A properly constructed and installed magnetic gas treatment unit will increase. Informal tests by Natural Energy Works personnel also confirm an increase in mileage, from about $5 \%$ to $15 \%$ depending upon the type of vehicle, environmental conditions and driver.

Similar to water, magnetic treatment alters the phase-change behavior of gasoline. Some theories propos that magnetic treatment increases the gasoline vaporization allowing a more dispersed and mixed air/fuel mixture. There are other theories proposing that the electrical ionization of gas droplets is affected or fuel fluid is activated or charged and gains special characteristics. Some field experiments indicated that magnetic treatment of fuel can increase the combustion ratio to reduce both fuel consumption and air pollution. The magnets can be used on standard carbureted or fuel-injected gasoline or diesel engines in different vehicles.

\section{CONCLUSION}

MWT is a relatively new technique in environmental management. Magnetic water technology has shown great potentials in different fields of environmental management. Scale prevention/elimination, soil enhancement, plant growth, crop yield, water saving, and wastewater treatment are some of these applications. Magnetic treatment of water restructures the water molecules into tiny, uniform and hexagonally structured cluster easing their travel through the passageways in plant and animal cell membranes. In addition, toxic agents cannot enter the MW structure. These features make MW a bio-friendly compound for plant and animal cells. For developing efficient techniques in the these fields controlled laboratory experiments followed by field studies are needed to shed light on the mechanism of actions of the MWT technique.

\section{REFERENCES}

1. Yadollahpour, A., et al., Repetitive transcranial magnetic stimulation decreases the kindling induced synaptic potentiation: effects of frequency and coil shape. Epilepsy Res, 108(2): p. 190-201 (2014).

2. Yadollahpour, A. and M. Jalilifar, Electromagnetic Fields in the Treatment of Wound:A Review of Current Techniques and Future Perspective. J Pure Appl Microbio, 8(4): p. 2863-2877 (2014).

3. Yadollahpour, A., M. Jalilifar, and S. Rashidi, Antimicrobial Effects of Electromagnetic Fields: A Review of Current Techniques and Mechanisms of Action. J Pure Appl Microbio, 8(5): p. 4031-4043 (2014).

4. Yadollahpour, A. and S. Rashidi, Therapeutic Applications of Electromagnetic Fields in Musculoskeletal Disorders: A Review of Current Techniques and Mechanisms of Action. Biomedical and Pharmacology Journal, 7(1): p. 23-32 (2014).

5. Yadollahpour, A. and S. Rashidi, A Review of
Electromagnetic Field Based Treatments for Different Bone Fractures. Biosci., Biotech. Res. Asia, 11(2): p. 611-620 (2014).

6. Yadollahpour, A., et al., Electromagnetic Fields for the Treatments of Wastewater: A Review of Applications and Future Opportunities. J Pure Appl Microbio, 8(5): p. 3711-3719 (2014).

7. Yadollahpour, A. and Z. Rezaee, Electroporation as a New Cancer Treatment Technique: A Review on the Mechanisms of Action. Biomedical \& Pharmacology Journal, 7(1): p. 53-62 (2014).

8. Gholizadeh, M. and H. Arabshahi, The effect of magnetic water on growth and quality improvement of poultry. Middle-East Journal of Scientific Research, 3 (2008).

9. Plantje, M.m.m.j.n., The Effects Of Magnet Treated Irrigation Water On Kentucky Bluegrass In A Greenhouse Environment.

10. Duarte Diaz, C., et al., Effects of magnetic treatment of irrigation water on the tomato crop. Hortic. Abst, 69: p. 494 (1997). 
11. Clark, J.P., Processing-Pulsed Electric Field Processing. Food Technology-Chicago, 60(1): p. 66-67 (2006).

12. Góngora-Nieto, M., et al., Food processing by pulsed electric fields: treatment delivery, inactivation level, and regulatory aspects. LWT-Food Science and Technology, 35(5): p. 375-388 (2002).

13. Kempkes, M., et al., Pulsed electric field (PEF) systems for commercial food and juice processing. Case studies in novel food processing technologies: innovations in processing, packaging, and predictive modelling, 2010: p. 73-102.

14. Min, S., et al., Commercial Scale Pulsed Electric Field Processing of Orange Juice. Journal of Food Science, 68(4): p. 1265-1271 (2003).

15. Min, S., Z.T. Jin, and Q.H. Zhang, Commercial scale pulsed electric field processing of tomato juice. Journal of agricultural and food chemistry, 51(11): p. 3338-3344 (2003).

16. Bolto, B.A., Magnetic particle technology for wastewater treatment. Waste management, 10(1): p. 11-21 (1990).

17. Yavuz, H. and S.S. Celebi, Effects of magnetic field on activity of activated sludge in wastewater treatment. Enzyme and Microbial Technology, 26(1): p. 22-27 (2000).

18. Parsons, S.A., et al., Magnetic treatment of calcium carbonate scale-effect of $\mathrm{pH}$ control. Water Research, 31(2): p. 339-342 (1997).

19. Schwartz, P. and W. Klassen, Estimate of losses caused by insects and mites to agricultural crops [in the US]. CRC Handbook of pest management in Agriculture, 1981.

20. Balouchi, H., A. Seyed, and B. Mahdavi, Electromagnetic Field Influence on Annual Medics, Barley, Dodder and Barnyard Grass Seed Germination. Pakistan Journal of Biological Sciences, 1: p. 1-6 (2007).

21. Moon, J.-D. and H.-S. Chung, Acceleration of germination of tomato seed by applying $\mathrm{AC}$ electric and magnetic fields. Journal of electrostatics, 48(2): p. 103-114 (2000).

22. Flórez, M., M.V. Carbonell, and E. Martínez, Exposure of maize seeds to stationary magnetic fields: Effects on germination and early growth. Environmental and Experimental Botany, 59(1): p. 68-75 (2007).
23. Kronenberg, K., MAGNETIZED II. Aqua Magazine, Sep, 1993.

24. Florestano, E., J. Marchello, and S. Bhat, Magnetic water treatment in lieu of chemicals. Chemical Engineering World, 31: p. 133-140 (1996).

25. Bogatin, J., et al., Magnetic treatment of irrigation water: experimental results and application conditions. Environmental science \& technology, 1999. 33(8): p. 1280-1285.

26. Hilal, M. and M. Hilal, Application of magnetic technologies in desert agriculture. I-Seed germination and seedling emergence of some crops in a saline calcareous soil. Egyptian Journal of Soil Science, 40(3): p. 413-422 (2000).

27. Aladjadjiyan, A., Study of the influence of magnetic field on some biological characteristics of Zea mais. Journal of Central European Agriculture, 3(2) (2002).

28. Cuartero, J. and R. Fernández-Muñoz, Tomato and salinity. Scientia Horticulturae, 78(1): p. 83-125 (1998).

29. Ruzic, R. and I. Jerman, Weak magnetic field decreases heat stress in cress seedlings. Electromagnetic Biology and Medicine, 21(1): p. 69-80 (2002).

30. Rutkowska-Naro ¿niak, A., Zastosowanie stae ${ }^{3}$ o pola magnetycznego do intensyfikacji biodegradacji zanieczyszczeñ w œciekach. Praca doktorska, Warszawa, 1997.

31. Rochalska, M., Improving of seeds quality with the frequent magnetic field. Part. I. Laboratory experiments. Biuletyn-instytutu Hodowli I Aklimatyzacji Roslin, : p. 61-76 (2001).

32. Reimers, R.S., T.G. Akers, and L. White, Use of applied fields in biological treatment of toxic substances, wastewater, and sludges. Adv Biotechnol Processes, 12: p. 235-72 (1989).

33. Pittman, U., Effects of magnetic seed treatment on yields of barley, wheat, and oats in southern Alberta. Canadian Journal of Plant Science, . 57(1): p. 37-45 (1977).

34. Ozdemir, S., O.H. Dede, and G. Koseoglu, Electromagnetic water treatment and water quality effect on germination, rooting and plant growth on flower. Asian Journal of Water, Environment and Pollution, 2(2): p. 9-13 (2005). 
35. Ozaki, H., Z. Liu, and Y. Terashima, Utilization of microorganisms immobilized with magnetic particles for sewage and wastewater treatment. Water Science \& Technology, 23(4-6): p. 1125-1136 (1991).

36. Novitsky, Y.I., et al., Growth of green onions in a weak permanent magnetic field. Russian Journal of Plant Physiology, 48(6): p. 709-716 (2001).

37. Kozic, V. and L. Lipus, Magnetic water treatment for a less tenacious scale. Journal of chemical information and computer sciences, 43(6): p. 1815-1819 (2003).

38. Jung, J. and S. Sofer, Enhancement of phenol biodegradation by south magnetic field exposure. Journal of chemical technology and biotechnology, 70(3): p. 299-303 (1997).

39. Jung, J., et al., Biodegradation of phenol: a comparative study with and without applying magnetic fields. Journal of Chemical Technology and Biotechnology, 56(1): p. 7376 (1993).

40. Yeoman, S., et al., The removal of phosphorus during wastewater treatment: a review. Environmental Pollution, 49(3): p. 183-233 (1988).

41. Tchobanoglous, G. and F.L. Burton, Wastewater engineering-treatment disposal, and reuse. revised. 1991.

42. Spellman, F.R., Handbook of water and wastewater treatment plant operations. 2013: CRC Press.

43. Liu, Y., et al., Effects of an electric field and zero valent iron on anaerobic treatment of azo dye wastewater and microbial community structures. Bioresour Technol, 102(3): p. 2578-84 (2011).

44. Ji, Y., et al., Enhancement of biological treatment of wastewater by magnetic field. Bioresour Technol, 101(22): p. 8535-40 (2010).

45. Cooper, P., T. Dee, and G. Yang. Nutrient removal-methods of meeting the EC urban wastewater directive. in Fourth Annual Conference on Industrial Wastewater Treatment, Esher, Surrey. 1993.

46. Krzemieniewski, M., et al., The influence of different intensity electromagnetic fields on phosphorus and COD removal from domestic wastewater in steel packing systems. Polish
Journal of Environmental Studies, 13(4): p. 381-387 (2004).

47. £ebkowska, M., Wp ${ }^{3} y w$ sta ${ }^{3}$ ego pola magnetycznego na biodegradacjê zwi'zków organicznych. 1991:Wydawnictwa Politechniki Warszawskiej.

48. Dini, L. and L. Abbro, Bioeffects of moderateintensity static magnetic fields on cell cultures. Micron, 36(3): p. 195-217 (2005).

49. Rosen, A.D., Membrane response to static magnetic fields: effect of exposure duration. Biochimica et Biophysica Acta (BBA)Biomembranes, 1148(2): p. 317-320 (1993).

50. Rosen, A.D., Threshold and limits of magnetic field action at the presynaptic membrane. Biochimica et Biophysica Acta (BBA)Biomembranes, 1193(1): p. 62-66 (1994).

51. Rosen, A.D., Inhibition of calcium channel activation in $\mathrm{GH} 3$ cells by static magnetic fields. Biochimica et Biophysica Acta (BBA)Biomembranes, 1282(1): p. 149-155 (1996).

52. Rosen, A.D., Effect of a $125 \mathrm{mT}$ static magnetic field on the kinetics of voltage activated $\mathrm{Na}+$ channels in $\mathrm{GH} 3$ cells. Bioelectromagnetics, . 24(7): p. 517-523 (2003).

53. Strašák, L., V.r. Vetterl, and J. Šmarda, Effects of low-frequency magnetic fields on bacteria< i> Escherichia coli</i>. Bioelectrochemistry, 55(1): p. 161-164 (2002).

54. Lin, I.J. and J. Yotvat, Magnetic treatment of water used for agricultural purposes. 1991, Google Patents.

55. Qi, D., et al., Simple approach to wafer-scale self-cleaning antireflective silicon surfaces. Langmuir, 25(14): p. 7769-7772 (2009).

56. Cort, S.L., Methods for removing heavy metals from water using chemical precipitation and field separation methods. 2005, Google Patents.

57. Busch, K.W. and M.A. Busch, Laborator studies on magnetic water treatment and their relationship to a possible mechanism for scale reduction. Desalination, 109(2): $p$. 131-148 (1997).

58. Mostafazadeh-Fard, B., et al., Effects of magnetized water and irrigation water salinity on soil moisture distribution in trickle irrigation. Journal of Irrigation and Drainage Engineering, 137(6): p. 398-402 (2010).

59. Wang, Y., et al., Rapid onset of calcium 
carbonate crystallization under the influence of a magnetic field. Water Research, 31(2): p. 346-350 (1997).

60. Powell, M.R., Magnetic Water and Fuel Treatment: Myth, Magic, or Mainstream Science? Skeptical Inquirer, 22: p. 27-31
(1998).

61. Hasson, D. and D. Bramson, Effectiveness of magnetic water treatment in suppressing calcium carbonate scale deposition. Industrial \& Engineering Chemistry Process Design and Development, 24(3): p. 588-592 (1985). 